\title{
Cancer Education
}

\section{Development of Oncology Nursing in Nepal: Historical Perspective}

\author{
Sarojini Sharma \\ B. P. Koirala Memorial Cancer Hospital Nursing College, Chitwan, Nepal
}

\begin{abstract}
Professional nursing in general and oncology nursing in particular underwent significant changes and a distinct paradigm shift in oncology nursing education and practice in the 30 year period of time (1991 to 2020), which promoted the advancement of oncology nursing. This historical review and reflection provides lessons for contemporary clinical cancer nurses, researchers, policy makers and educators to approach the nursing issues, necessity of cooperation/ collaboration and maintaining the passion for increasingly complex nursing speciality.
\end{abstract}

Keywords: History, Nepal, Nursing.

\section{Introduction}

The history of cancer treatment in Nepal is very short. Bir Hospital is the first center in Nepal which served as a central institution for cancer treatment until other cancer centers were established in the country. Bir Hospital is one of the oldest and the biggest government general hospital in Nepal which is located in Kathmandu. Primarily, only chemotherapy and surgery were available. The new era of cancer treatment with radiotherapy began only in the past three decades when the first telecobalt radiotherapy unit in the country was installed. This was in 1991 in the Bir Hospital in cooperation with the Indian government. In the beginning, the major seven hospitals dealing with cancer patients in Nepal are: 1. B. P. Koirala Memorial Cancer Hospital, Bharatpur, 2. Bir Hospital/ National Academy of Medical Sciences (NAMS), Kathmandu, 3. Teaching Hospital, Tribhuwan University, Kathmandu, 4. Kanti Children's Hospital, Kathmandu, 5. Bhaktapur Cancer Hospital, Bhaktapur, 6. B. P. Koirala Institutes of Health Sciences, Dharan, 7. Manipal College of Medical Sciences, Pokhara. Only 5 of them had radiotherapy facilities. At present, many private hospitals started multimodal cancer treatment not only in the Kathmandu Valley but also outside the valley from east to west of the country. ${ }^{1}$
Patients from all parts of the country visit these hospitals for cancer treatment. Cancer treatment worldwide is very costly requiring expensive facilities, highly specialized health care personnel, and expensive drugs. Nepal is still struggling to improve and manage even conventional modalities for cancer management. Gradual progress has been made in cancer management including treatment, prevention, and control in Nepal despite the poor socioeconomical and unstable political condition of the country.

Nursing has undergone a dramatic change in response to social needs and influences. Nursing, at the present, is different from nursing as it was practiced years ago, and it is expanded to continue changing during the 21 st century. Today, nursing is a profession with rich career opportunities that change and reflect the society in which nurse lives. Advancement in treatment of disease and specialty/ subspecialty in medicine demands the specialty in nursing education and practice like oncology nurse, midwife, nurse practitioner, nurse specialist in cardiac care, nephrology care, etc.

The history of oncology nursing in Nepal is too short. After the establishment of the cancer hospitals, nurses

\section{Correspondence}

Sarojini Sharma, B. P. Koirala Memorial Cancer Hospital Nursing College, Chitwan, Nepal sarojini.josi@gmail.com 
realized their different roles and functions in the cancer centers. For the first time, Ms. Daya Laxmi Vaidya went to Australia to get a degree in oncology nursing. Many foreigner nurses having oncology experience from Canada, UK, Japan, and America came to Nepal and volunteered in different cancer hospitals of Nepal in different points of time and helped Nepalese nurses to get an insight about oncology nursing. Gradually, nurses working in cancer hospitals got the opportunity to have fellowship in world-renowned cancer hospitals, supported by UICC. ${ }^{2}$

To identify the critical elements of oncology nursing and a major shift in practice and education. Annual report of cancer hospitals, nursing history literature, archival materials in the library of the cancer hospital, journals and personal communication.

\section{Advances in Cancer Treatment and Nursing Practice \\ Radiation Oncology and Nursing}

Radiotherapy is one of the main types of cancer treatment modality. It uses ionizing radiation to destroy cancer cells and limit cell growth. High doses of radiation are used to kill cancer cells and shrink tumors. Radiation therapy treats cancer alone or in combination with chemotherapy or before/after surgery. Ionizing radiation either from radiation generator or radioactive materials are used for radiation therapy. Electrons, gamma rays, protons, etc are the most common radiations used for radiotherapy.

In Nepal, Radiation therapy service was started in 1991 in Bir Hospital, Kathmandu using a Cobalt radiation teletherapy machine. In 1998, Bhaktapur Cancer Hospital also started cancer patient treatment from the Co-60 machine. B. P. Koirala Memorial Cancer Hospital (BPKMCH) had installed a cobalt teletherapy machine in 1999 and two linear accelerators and a high dose rate brachytherapy machine in 2002. In almost 30 years journey of radiation therapy, a few other radiotherapy centers in the private sector have started the radiotherapy services, Kathmandu Cancer Center in mid-2016, Nepal Cancer Hospital and Research Center in late 2016, and Purbanchal Cancer Hospital in early 2020. Five linear accelerators, two cobalt-60 units are on-service in 6 radiotherapy centers. Most installed linear accelerators are high-end radiotherapy machine with an on-board imager that helps to deliver a precise and accurate radiation dose to the target. Hence, cancer patients in Nepal can take benefit of modern RT techniques like IMRT, IGRT, VMAT, and SBRT advanced treatment modalities which are available in public as well as private centers. $^{3}$

Nurses role in cancer treatment are in two aspects, one being technical and other being psychological. In the beginning, nurses' responsibilities associated with radiation therapy included attending to patient's emotional reactions, identification of gaps in the patient's knowledge, correction of misconceptions, and reinforcement of information provided by the physicians. Nurses were expected to provide assistance in maintaining a constant field with consistent positioning during the treatment and to ensure accurate dosage. In 1997/1998, BPKMCH sent a few nurses to TATA Memorial Hospital, India for observation training related to administration of the cytotoxic agent and care of the patient having radiation therapy. Those experiences and exposure generated new insight into the nursing care of the patient undergoing radiation therapy.

At present, nurses have additional and definite responsibilities in brachytherapy; instructing, monitoring patient's hygiene, applicator positioning, management of emesis, special diet, prevention of irritation and infection in the treated area. Nurses administer the liver extract, vitamin $\mathrm{B}$, and other substances for the treatment of side effects of radiation therapy: nausea, anorexia, fatigue, malaise, diarrhea, etc, which occur more frequently when the gastrointestinal tract fell within the field of radiation treatment. In radiation therapy, the nurses play an important role in patient education regarding side effect management, home care, compliance on the treatment, psychological perspectives and follow-up.

\section{Surgical Oncology and Nursing}

Peri-operative nursing care of cancer patients depends on the type of cancer, type of surgical risk, type of surgery, neoadjuvant therapy, and pre-existing physiologic defects. Surgery over irradiated tissue increases the risk of post-op infection, wound dehiscence, and necrosis. There are fibrosis and obliteration of lymphatic and vascular channels. Similarly, some cytotoxic agents (Methotraxate, Cyclophosphafamide, Flurouracil, Doxorubicin) reduce the tensile strength of surgical 
wound while some chemo drugs have severe toxicity to specific organ. ${ }^{4}$

Roles of nurses in surgical oncology setting are being recognized by the institutions, with the advancement in technology, radical nature of the surgery, organ amputation, and disfiguring surgery, reconstructive surgery is demanding more specialized nursing care. Nursing care for breast cancer patients focused on lymphedema prevention and its management. Stoma creating surgery emphasized the care of stoma, prevention of peristomal skin complications, foul order control, leakage control, appropriate use of ostomy appliances, and colostomy irrigation $^{5}$. The role of enterostomal therapist (ET) was recognized by Norma Gill and Dr. Turnbull in 1954, then in 1961 opened the school for enterostomal therapist in Cleveland clinic to increase the number of enterostomal therapist. In contrary, these specialist job was very effort consuming due to unavailability of different appliances and modern dressing materials in our part of the world which has demotivated those nurses to carry their jobs. ${ }^{5}$

Another important role of the nurse is as a counselor, especially for the patient who underwent stomy surgery, vulvectomy, penectomy, limb amputation, mastectomy, and other physically disfiguring surgical procedure. It was found that dealing with a patient who lost the selfrespect and body image is very tough. Till date, the role of psychologist/counselor in cancer hospital is not emphasized thus, nurses are responsible for counseling and providing psychological support to the patients and their families. ${ }^{7}$

\section{Chemotherapy and Nursing}

Till 1960, the administration of fluids and nutrients via IV as a nursing function was controversial. Venipuncture was strictly the physician's domain. During World War II, nurses began doing vein puncture on the front lines and in-home, hospitals where military service had depleted the physician population. Nurse's role in administration and management of cytotoxic chemotherapy in the 20th and 21st centuries evolved from the long and heated debates initiated during World War II and continuing up to the $1960 .^{8}$

At the beginning of Oncology service (1991), Cytotoxic medicines were administered in seven major hospitals in Nepal. Nurses were not formally trained however, they administered cytotoxic drugs under the guidance of a physician. There was no biosafety cabinet for chemo preparation. Nurses prepared the cytotoxic drugs at the bedside and administered without proper safety precautions. For the first time, BPKMCH brought the Biosafety cabinet with laminar airflows, which assured the nurses' safety from cytotoxic agents during preparation time. Nurses were involved in the preparation and administration of cytotoxic agents. Further responsibilities associated with chemotherapy included, pre-chemo counseling, collection of cytotoxic drugs, preparation/mixing/dissolving, administration, safe disposal of cytotoxic waste, spill management, extravasation management and side effects management of cytotoxic drugs. ${ }^{7}$

Over time technological advances changed the nurses' role. Nurses were allowed to insert the peripherally inserted central catheter ( PICC). PICC post-insertion care of catheter and wound care of the exit site was the major task of the nurses observed in the chemo unit. Chemo administration through the port and port care was another responsibility of the nurses working in the chemotherapy unit. ${ }^{9}$

Very few nurses working in chemotherapy units in both the government and the private hospitals were formally trained. Most of them were experience based trainees trained by seniors in same unit (Skill transfer) and some were trained in some other chemo units within the country.

Nowadays, most of the Medical colleges and private hospitals of Nepal also have separate oncology unit where cytotoxic agents are administered.

\section{Cancer Prevention and Control and Nursing}

Prevention is better than cure. Cancer is assumed to be preventable and controllable if it is detected earlier. Formally, role of nursing in cancer control did not emerge until the 1970s. Linda White and her colleagues at the University of Texas MD Anderson Hospital created the first structured institutional program to teach nurses how to do cancer screening and detection in $1975^{10}$, and efforts to demonstrate and build nurse's competencies in cancer control continue to present date. Advances in prevention and early detection included the promotion of breast self-examination and the implementation of 
the Papanicolaou smear technique to identify early cancerous changes in the cervix.

Nurses are key in case finding positions because they work with patients and have the opportunity to observe signs and apply knowledge in discerning cancer in such areas as the skin, breast, cervix, oral cavity, where the disease frequently occurs. Rosalie Peterson 1954 noted the importance of nurses' knowledge and attitude in cancer control activities. ${ }^{11}$

In Nepal, the nurse's role in cancer prevention and early detection was not realized before 2010. Very few Government (Govt.) hospital had cervical cancer screening outpatient department(OPD). Usually, the gynecologist was involved in taking the sample for Pap Test. Nurses were in an assistant role. Cervical Cancer screening in a community setting and mass scale was not the priority program of Govt. After the establishment of the cancer hospital, it established the screening clinic/OPD and started screening activities as per the institutions' mission. In 2010, Govt. of Nepal developed the "National Guideline for cervical cancer screening and prevention". The policy has identified the primary health care centers as the starting point of screening. Based on the guidelines, the training package was developed about visual inspection with acetic acid (VIA) and target trainee were the nurses working in the primary health care center. ${ }^{12}$

B.P. Koirala Memorial Cancer Hospital had a separate department of cancer prevention, control, and research, and the department is led by the public health officer and nurses. The hospital started cancer prevention and control activities not only in hospital OPD but also in community settings. The main activities of the preventive program are the VIA test, teaching self-breast examination/ performing clinical breast examination, and oral examination. Nurses have a vital role in cancer screening activities. ${ }^{13}$

General Governmental hospitals, Maternity Hospital, and private cancer hospitals are also running cancer screening program as per the institutional policy and nurses have an important role in the screening program.

\section{Palliative Care and Nursing}

The impetus for the development of palliative care in Nepal was based on the recognition by a group of Nepali oncologist and Nurses. There have been various interactions with experts from other countries, the collaboration between the International Network for Cancer Treatment and Research (INCTR) and the Nepal Palliative care group prepare the concept of palliative care guidelines. ${ }^{14}$

Palliative care was institutionalized after 2000 AD when the formal institution (Hospice Nepal) opened for the people living with advanced disease especially cancer with 10-bed facilities. Afterward, different governmental and non-governmental institutions started to cater to the palliative needs of the patients. Namely, the following institutions started palliative care services; B. P. Koirala Memorial Cancer Hospital started palliative care services since 2004, Bhaktapur Cancer Hospital also started to provide palliative care services with separate wards. Scheer Memorial Hospital has also developed a dedicated unit for palliative care since 2005. Kanti Children Hospital also has a dedicated oncology unit. Within the oncology unit, there is a separate bed for the children having needs for palliative care. Nepal Cancer Hospital and Research Center, Harisiddhi is also providing palliative care to the patients. Nepal Hospice and Palliative Care by Binaytara Foundation started in 2016 with the objective of home hospice and palliative care. So like, many other organizations are catering palliative care needs of the patients. ${ }^{15}$

A multidimensional professional team is required for palliative care. Nurses are the pillar of the palliative care team because they serve 24 hours a day and 7 days a week. Nurse's responsibilities associated with palliative were found to be; pain assessment and pain management, administration of opioid drugs, fistula management, dyspnea management, constipation management, bleeding management, difficult wound management, psychological and spiritual support, breaking bad news, etc.

In the beginning, nurses working in the above-mentioned institutions were not trained, even then they were taking care of patients based on general nursing knowledge. Institutions realized the need for training for those who were working in a palliative setting, thereafter, they were sent for palliative care training aboard mainly in India, Canada, and the United Kingdom but the number of nurses were very few. Since 2013AD, Nepal govt. in association with the Nepalese Association of Palliative care started 15 days palliative care training for doctors, nurses, and paramedics regularly. After that majority of 
nurses working in palliative care settings are trained in Palliative care..$^{15,16}$

\section{The Shift from Hospital to Home}

The demand for home care is gradually increasing in Nepal, especially in the terminal phase of cancer. Till today Nepal govt. has no policy, rules, and guidelines for home care nursing. Very few cancer hospitals have started home care services in the form of the home visit and consultation services as per the convenience of the institute. BPKMCH had started its home visit service since 2010 with a multidisciplinary team and nurses were key to that team. Private hospice and palliative care centers gradually showing interest in-home care and have started their services in their catchment areas. The shift in site of care from hospital to home provided an early indication of the need for teamwork: the collaboration among physicians, families, and social worker. ${ }^{21}$

\section{Oncology Nursing Education}

The history of nursing education in Nepal is described in two phases. The first phase was before 1972. At that time nursing education was not under the control of the university. Nursing schools and training were regulated by the ministry of health and health division. The second phase was after 1972 when the new education policy was introduced by the government and all nursing schools were placed under the university (TU, IOM) and nursing courses were academically accredited. Bachelor of nursing was introduced in 1977 (2033) with midwifery major subject. Gradually, other tracts were introduced namely; adult health nursing, pediatric health nursing, community health nursing, psychiatric nursing, etc. In 1995, the postgraduate course was started with 5 students. Successively, the stream was extended and the number of students was increased. In 2005, B. P. Koirala Institute of Health Sciences started 4 years B. Sc Nursing Course. Another milestone in nursing education was in 2011, Maharajgunj Nursing Campus in Kathmandu started Ph.D. in nursing. After the restoration of democracy in 1991, National health policy opened the door for the private sector, as a result, there has been a massive increase in the privatization of nursing education from the PCL level to master level under different universities. ${ }^{17}$

Till 2004, the Oncology nursing (Speciality) course was not offered by the existing universities of Nepal. Nurses used to work in a cancer hospital with general nursing knowledge. Kathmandu University for the first time offered the oncology elective course for B. Sc. Nursing students as an elective course with 6 credit hours (100 full marks). Students were posted to different cancer hospitals in Nepal for skill development. Another important milestone of oncology nursing education was the Purvanchal University's Post Basic Bachelor Nursing program, where they incorporated the oncology course as a core course in their curriculum in 2013/2014 with 3 credit hours (50 full marks). Institute of Medicine also revised the nursing curriculum and incorporated the oncology issues in the system-wise disease process. Council for technical education and Vocational training (CTEVT) also incorporated the separate oncology chapter for PCL students by the year 2012/2013 Curriculum. ${ }^{18,19}$

To bridge the gap of oncology nursing education, B. P. Koirala Memorial Cancer Hospital (BPKMCH), the nursing department started the 3 months basic oncology nursing training within the hospital in year 2012. Nurses from different hospitals and colleges participated in the training. Similarly, registered nurses working in different cancer hospitals were also posted for short term ( 2 weeks to 4 weeks) to $\mathrm{BPKMCH}$ for practical experience and skill development. BPKMCH, the nursing department published the first edition of the nursing manual in the year 2010 and was updated to 3rd edition in $2017^{4}$, which is available in Nepal. The manual has been used by the nurses working in oncology setting and educators have relied on manual as a textbook. December 22nd, 2018, became the historical day for the oncology nursing fraternity, as B. P. Koirala Memorial Cancer Hospital opened the Nursing College as a joint constituent program of Pokhara University. ${ }^{22}$ Proficiency Certificate Level (PCL) graduates with one year experience can apply for the Bachelor of Nursing Science (BNS Oncology) program. University offered the 3 years BNS course with oncology specialty. Twenty students enrolled in the first batch. $^{20}$

\section{Conclusion}

Professional nursing in general and oncology nursing, in particular, underwent significant changes and a distinct paradigm shift in cancer nursing education and practice in the 30 years (1991 to 2020), which promoted the advancement of cancer nursing. 


\section{References}

1. B. P. Koirala Memorial Cancer Hospital, Cancer Prevention, Control \& Research Department. National Cancer Registry Program; 2017.

2. B. P. Koirala Memorial Cancer Hospital, Annual Report 2005.

3. Subedi KS, Sharma P.Cancer Treatment in Nepal: A historical background, development of treatment facilities, epidemiology, and challenges for prevention and control of Cancer.Asain Journal of Cancer 2012; 11( 3):205-212.

4. B. P. Koirala Memorial Cancer Hospital Oncology Nursing Manual. 3rd edition 2017.

5. Sharma S, Subedi B. Enterostomal Therapy Service: Ten Year Experience at B.P. Koirala Memorial Cancer Hospital. Nepalese Journal of Cancer 2019, 3(1):3945.

6. Blakely P. Future trends in ET Nursing Internationally. In Erwin-Toth, Krasner D, Editors: Enterostomal Therapy Nursing growth and evolution of a Nursing specialty world wide. Baltimore1996, Halgo.

7. B. P. Koirala Memorial Cancer Hospital. Annual Report 2013.

8. Pamela J. Haylock. Cancer Nursing: The Modern Era.Oncology Nursing Forum. Supplement 2011; 38(6): e7- e14.

9. B. P. Koirala Memorial Cancer Hospital. Annual Report 2018.

10. Hilkemeyer, R. A Historical perspective in Cancer Nursing. Oncology Nursing Forum1985;12(1),6-15.

11. Kottke, T.E\& Trapp, M.A. The quality of pap test specimens collected by nurses in breast and cervical cancer screening Clinic. American Journal of Preventive Medicine1998,14, 196-200.

12. Cervical Cancer Screening and Prevention Project in Nepal. Retrive on http// karunanepal.org $>$ ccsp 13th august 2020.

13. B. P. Koirala Memorial Cancer Hospital. Annual Report 2014.

14. Munday \& Powys. Reflection on the Opportunities \& Challenges for Palliative care in Nepal. Christina Journal for Global Health 2017; 4(3): 12-20.

15. Brown S, Vaidya P, RSW, MSW (C) et al. Palliative Care Development: The Nepal Model. Journal of Pain and Symptom Management 2007; 33(5):573577.

16. B. P. Koirala Memorial Cancer Hospital. Annual Report 2017.

17. Satyam P, Yadav P, Yadav K. Perspectives of Developing Nursing Education in Nepal. Nurse Care Open Access J. 2018; 5(4): 214-220.

18. Kathmandu University. Curriculum for Bachelor of Science in Nursing. 2004.

19. Purvanchal University. Curriculum for Bachelor in Nursing Science 2013/2014.

20. Pokhara University, Faculty of Health Sciences. Curriculum for Bachelor of Nursing Science 2018.

21. B. P. Koirala Memorial Cancer Hospital. Annual Report 2010.

22. B. P. Koirala Memorial Cancer Hospital. Annual Report 2018. 